\title{
非线性泛函差分系统的吸引域
}

\section{徐道义 徐安石}

(四川联合大学数学系, 成都 610064)

摘要 讨论了具有无穷时滞的泛函差分系统的吸引域, 利用谱半径 $\rho$ 小于 1 的含参非负矩阵 及属于 $\rho$ 的特征子空间给出了确定这类系统吸引域的充分条件.

\section{关键词 泛函差分方程 吸引域 稳定性}

由于非线性系统的平衡点一般不唯一, 故在已有的结果中, 对这类系统稳定性的讨论往往 是局部的 ${ }^{[1 ~ 3]}$. 就是经典的 Lyapunov 渐近稳定性定义给出的吸引域也可能充分小. 因此确 定吸引域的大小一直是人们关注的问题. 本文则将对这一问题进行研究, 给出确定非线性泛 函差分系统吸引域的具体方法.

考虑具有无穷时滞的泛函差分系统

$$
x(m+1)=f\left(m, x_{m}\right), \quad m \in \mathrm{Z},
$$

其中 $x \in \mathrm{R}^{n}, f: \mathrm{Z} \times C^{\rightarrow} \mathrm{R}^{n}, f(m, 0) \equiv 0$ 保证方程(1) 总有零解, $\mathrm{Z}$ 是整数集, $C$ 是所有函 数 $\varphi: \mathrm{Z}^{-} \rightarrow \mathrm{R}^{n}$ 构成的集合, $\mathrm{Z}^{-}=\{x \mid x \in \mathrm{Z}, x \leqslant 0\}, x_{m}(s)=x(m+s), \forall s \in \mathrm{Z}^{-}$. 所以 $x_{m}$ $\in C, \forall m \in Z$. 对于任意 $m_{0} \in \mathrm{Z}^{+}=\{x \mid x \in \mathrm{Z}, x \geqslant 0\}, \varphi \in C$, 设方程(1) 满足初始条件 $x_{m_{0}}$ $=\varphi\left(m \leqslant m_{0}\right)$ 的解存在, 并记作 $x(m)=x\left(m, m_{0}, \varphi\right)$.

令 $[\cdot]^{+}$为绝对值算子, 如 $x \in \mathrm{R}^{n}$, 则 $[x]^{+}$表示对 $x$ 的每一个元素取绝对值而形成的向 量. 并定义 $x_{m}=\left[x_{1 m}, \cdots, x_{n m}\right]^{\mathrm{T}},\left[x_{m}\right]_{r}^{+}=\left[\left\|x_{1 m}\right\|_{r}, \cdots,\left\|x_{n m}\right\|_{r}\right]^{+}$, 其中

$$
\left\|x_{i m}\right\|_{r}=\sup _{r \leqslant 0}\left|x_{i}(m+s)\right|, \quad i=1, \cdots, n,
$$

这里 $r=r(m) \geqslant 0$. 当 $r=\infty$ 时约定 $\left[x_{m_{0}}\right]_{r}^{+}=[\varphi]_{\infty}^{+}$.

定义 1 若存在集合 $D \subset \mathrm{R}^{n}\left(D-\{0\}\right.$ 非空), 对于任意 $m_{0} \in \mathrm{Z}^{+}$及 $\varphi \in C$, 当 $[\varphi]_{\infty}^{+} \in D^{+}=$ $\{x \mid x \in D, x \geqslant 0\}$ 时都有

$$
\left[x\left(m, m_{0}, \varphi\right)\right]^{+} \leqslant[\varphi]_{\infty}^{+}, \forall m \geqslant m_{0},
$$

则称 $D$ 为 $(1)$ 的稳定域. 若还有

$$
\lim _{m} x\left(m, m_{0}, \varphi\right)=0,
$$

则称 $D$ 为方程 $(1)$ 的渐近稳定吸引域.

令 $P(A)$ 表示方阵 $A$ 的谱半径. 若 $A \geqslant 0$, 则 $P(A)$ 为 $A$ 的特征根(文献 [4], p. 503, 定理 8. 3. 1). 记 $w \rho(A)$ 为 $A$ 属于 $\rho(A)$ 的特征子空间, $w_{\rho}^{+}(A)=\{x \mid x \in w \rho(A), x \geqslant 0\} . A \leqslant B$ 表示矩阵 $A$ 的每一个元素都不超过 $B$ 的对应元素. $A<B$ 表示 $A \leqslant B$ 但 $A \neq B$.

定义 2 令 $\Omega \subset \mathrm{R}^{n}, A(x): \Omega^{\rightarrow} \mathrm{R}^{n \times n}$. 若对于任意 $x^{\prime}, x^{\prime \prime} \in \Omega$, 当 $x^{\prime} \leqslant x^{\prime \prime}$ 时有 $A\left(x^{\prime}\right) \leqslant$ $A\left(x^{\prime \prime}\right)$, 则称矩阵函数 $A(x)$ 在 $\Omega$ 内单调不减. 
定理 1 设有轴对称凸区域 $\Omega \subset \mathrm{R}^{n}$ 使得

$$
\left[f\left(m, x_{m}\right)\right]^{+} \leqslant A\left(\left[x_{m}\right]_{r}^{+}\right)\left[x_{m}\right]_{r}^{+}, \quad \forall x \in \Omega,
$$

其中 $A(s): \Omega^{+} \rightarrow \mathrm{R}^{n \times n}$ 在 $\Omega^{+}=\{x \mid x \in \Omega, x \geqslant 0\}$ 内单调不减, 若集合 $D-\{0\}$ 非空, 其中

$$
D=\left\{x \in \Omega \mid[x]^{+} \leqslant K \in \Omega^{+},[x]^{+} \in w^{+} \rho(A(K)), P(A(K))<1\right\},
$$

则 $\bar{D}$ (即式 $(5)$ 内 $P(A(K))<1$ 换为 $P(A(K)) \leqslant 1$ 而形成的集合) 为系统 $(1)$ 的稳定域. 若还 有 $\lim _{m}(m-r(m))=\infty$, 则 $D$ 为系统 $(1)$ 的渐近稳定吸引域.

证 先证 $\bar{D}$ 是系统(1) 的稳定域, 即

$$
[x(m)]^{+} \leqslant[\varphi]_{\infty}^{+}, \forall m \geqslant m_{0} .
$$

若不然, 必存在 $m^{*} \geqslant m_{0}$ 及某个 $i$ 使得

$$
\left|x_{i}\left(m^{*}+1\right)\right|>\left\|\varphi_{i}\right\|_{\infty} \text { 且 } \Gamma x(m) T^{+} \leqslant \Gamma \varphi T_{\infty}^{+}, \forall m \leqslant m^{*} .
$$

又因为 $\Omega$ 是轴对称凸域, 于是由式(7) 推得 $\left[x_{m}\right]_{r}^{+} \in \Omega^{+}\left(\forall m \leqslant m^{*}\right)$. 利用式(4)对系统 (1) 两端取绝对值算子得

$$
[x(m+1)]^{+} \leqslant A\left(\left[x_{m}\right]_{r}^{+}\right)\left[x_{m}\right]_{r}^{+}, \forall m \leqslant m^{*} .
$$

由 $A$ 的单调性及式(7) 的后一不等式得

$$
\left[x\left(m^{*}+1\right)\right]^{+} \leqslant A\left([\varphi]_{\infty}^{+}\right)[\varphi]_{\infty}^{+} \leqslant A(K)[\varphi]_{\infty}^{+} .
$$

因为 $[\varphi]_{\infty}^{+} \in w_{\rho}^{+}(A(K))$ 且 $P(A(K)) \leqslant 1$, 故

$$
\left[x\left(m^{*}+1\right)\right]^{+} \leqslant P(A(K))[\varphi]_{\infty}^{+} \leqslant[\varphi]_{\infty}^{+},
$$

这与式(7) 的前一不等式矛盾. 从而式(6) 成立且式(8) 对于所有 $m \in Z$ 成立. 于是有

$$
\Gamma x(m+1)\rceil^{+} \leqslant A(K)\left\lceil x_{m}\right]_{r}^{+}, \quad \forall m \in \mathrm{Z} .
$$

式(6)还保证了有常向量 $c \geqslant 0$ 使得 $\varlimsup_{m}[x(m)]^{+}=c \leqslant K$. 由于 $m-r(m) \rightarrow \infty(m \rightarrow \infty)$, 对 于任给的 $\varepsilon>0$, 必有充分大的 $M_{1}$ 使得 $m \geqslant M_{1}$ 时有

$$
\left[x_{m}\right]_{r}^{+} \leqslant c+\varepsilon, \quad e=[1, \cdots, 1]^{\mathrm{T}},
$$

也有 $M_{2} \geqslant M_{1}$ 使得 $\left[x\left(M_{2}+1\right)\right]^{+} \geqslant c-e \varepsilon$. 结合式(10)得

$$
c-\varepsilon \leqslant\left[x\left(M_{2}+1\right)\right]^{+} \leqslant A(K)\left[x_{M_{2}}\right]_{r}^{+} \leqslant A(K)(c+e \varepsilon) .
$$

因为在 $D$ 内 $\mathrm{P}(A(K))<1$, 从而 $[I-A(K)]^{-1} \geq 0$ (文献 $[3]$, p. 30). 那么由式(11) 推得

$$
c \leqslant[I-A(K)]^{-1}(I+A(K)) e \varepsilon \text {. }
$$

这就推得了 $\lim _{m} x(m)=0$. 即 $D$ 为系统 $(1)$ 的渐近稳定吸引域. 证毕.

注 1 本文方法也适合于有界时滞系统, 事实上, 令 $\mathrm{Z}_{h}^{-}=\left\{\mathrm{ZI} x \in \mathrm{Z}^{-}, x \geqslant-h, h \geqslant 0\right.$ 为整 数 $\}, C_{h}=\left\{\varphi \mid \varphi: \mathrm{Z}_{h}^{-} \rightarrow \mathrm{R}^{n}\right\}$, 将上面的 $C$ 换为 $C_{h},[\varphi]_{\infty}^{+}$换为 $[\varphi]_{h}^{+}$, 可以获得同样的证明.

例 1 考虑时滞差分系统

$$
x(m+1)=\frac{1}{\sqrt{2}}[\sin x(m-\tau)+\cos x(m-\tau)] x(m) \triangleq f\left(x_{m}\right),
$$

其中 $x \in \mathrm{R}, \tau$ 为非负整数.

取 $\Omega=\left|-\frac{\pi}{4}, \frac{\pi}{4}\right|$, 则对于任意 $x \in \Omega$ 都有 $|\sin x|=\sin |x|,|\cos x|=\cos |x|$. 故在 $\Omega$ 内总有 


$$
\left|f\left(x_{m}\right)\right| \leqslant \frac{1}{\sqrt{2}}\left(\sin \left|x_{m}\right| \tau+\cos \left|x_{m}\right| \tau\right)\left|x_{m}\right| \tau \triangleq A\left(\left|x_{m}\right| \tau\right)\left|x_{m}\right| \tau .
$$

这里 $A(s)$ 在 $\Omega^{+}$内单调不减(因为 $A^{\prime}(s) \geqslant 0, \forall s \in \Omega^{+}$), 并保证了

$$
A(K)=\frac{\sqrt{2}}{2}(\sin K+\cos K)<1, \forall K \in \Omega^{+} .
$$

由定理 1 , 系统(12) 的一个渐近稳定吸引域为

$$
D=\left|-\frac{\pi}{4}, \frac{\pi}{4}\right| \text {. }
$$

注 2 取 $\tau=0$, 系统( 12) 成为文献 [ 5] 例 2, 那里给出的吸引域为 $(-0.347,0.347)$, 这 里扩大为 $D$ 且无需将 $f$ 展成级数.

例 2 考虑具有无穷时滞的差分系统

$$
\mid \begin{aligned}
& x_{1}(m+1)=\frac{1}{4} x_{1}^{3}(m)+\frac{1}{9} x_{1}\left|m-\frac{2}{3} m\right| x_{2}^{2}\left|m-\frac{m}{2}\right| \triangleq f_{1}, \\
& x_{2}(m+1)=\frac{1}{4} x_{1}^{2}(m) x_{2}\left|m-\frac{2 m}{3}\right|+\frac{1}{9} x_{2}^{3}\left|m-\frac{m}{2}\right| \triangleq f_{2} .
\end{aligned}
$$

取 $r=\frac{m}{2}$, 显然 $m-r(m) \rightarrow \infty(m \rightarrow \infty)$ 且

$$
[f]^{+} \leqslant\left|\begin{array}{ccc}
\frac{1}{4}\left\|_{x_{1 m}}\right\|_{r}^{2} & \frac{1}{9}\left\|_{x_{1 m}}\right\|_{r}\left\|_{x_{2 m}}\right\|_{r} \\
\frac{1}{4}\left\|_{x_{1 m}}\right\|_{r}\left\|_{x_{2 m}}\right\|_{r} & \frac{1}{9}\left\|_{x_{2 m}}\right\|_{r}^{2}
\end{array}\right|\left|\begin{array}{c}
\left\|x_{1 m}\right\|_{r} \\
\left\|x_{2 m}\right\|_{r}
\end{array}\right|,
$$

从而

$$
\begin{gathered}
A(K)=\left|\begin{array}{cc}
\frac{1}{4} k_{1}^{2} & \frac{1}{9} k_{1} k_{2} \\
\frac{1}{4} k_{1} k_{2} & \frac{1}{9} k_{2}^{2}
\end{array}\right|, P(A(K))=\frac{1}{4} k_{1}^{2}+\frac{1}{9} k_{2}^{2}, \\
w^{+} \rho(A(K))=\left\{x \in \mathrm{R}^{2}\left|k_{2}\right| x_{1}\left|=k_{1}\right| x_{2} \mid\right\} .
\end{gathered}
$$

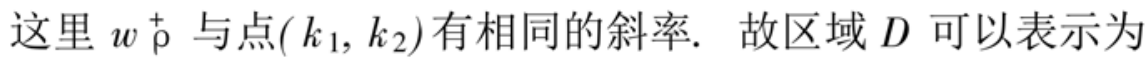

$$
D=\left|x \in \mathrm{R}^{2}\right|[x]^{+}<K, \frac{1}{4} k_{1}^{2}+\frac{1}{9} k_{2}^{2}<1|=| x \in \mathrm{R}^{2}\left|\frac{x_{1}^{2}}{4}+\frac{x_{2}^{2}}{9}<1\right| .
$$

由定理 1 , 系统 (14) 的一个稳定域为闭椭圆 $\bar{D}$, 渐近稳定域为 $D$.

注 3 本文方法也适用于泛函微分系统, 作者将另文讨论.

致谢 本工作为国家自然科学基金( 批准号: 19771059) 资助项目.

\section{参 考 文 献}

1 Lakshmikantham V, Trigiante D. Theory of Difference Equations. New York: Academic Press, 1988

2 Kelley W G, Peterson A C. Difference Equation. New York: Academic Press, 1991

3 Lasalle J P 著, 廖晓昕等译. 动力系统的稳定性. 武汉: 华中工学院出版社, 1983

4 Horn R A, Johnson C A. Matrix Analysis. Cambridge: Cambridge University Press, 1985 


\section{连续反馈混沌同步方式在保密通讯的中应用}

\section{张 宇 余俊明 杜功焕}

(南京大学声学研究所和近代声学国家重点实验室, 南京 210093)

摘要 利用Chua 混沌电路对连续反馈同步方式在保密通讯中的应用进行研究. 通过对混沌 同步原理的研究得出确保保密通讯得以实现的恢复同步系数 $K_{\mathrm{r}}>2$, 并利用 PSPICE 软件进 行仿真实验. 研究为避免系统分拆、任意信躁比下的保密通讯提供了理论和实验依据.

\section{关键词 保密通讯混沌同步 Chua 电路}

自从 Pecora 和 Carroll 提出他们的同步方案后 ${ }^{[1]}$, 国际上对混沌同步的理论和实验的研究 迅速开展起来, 混沌同步的应用也日益引起人们的极大关注, 并已成为混沌研究的前沿课题和 发展方向之一. 与混沌同步相关的最热门的论题是混沌同步技术在保密通讯中的应用.

Kocarev 等人 ${ }^{[2]}$ 及 Cuomo 等人 ${ }^{[3]}$ 在实验上先后利用 Pecora_Carroll 同步技术进行保密通 讯的研究, 然而他们的方法要求混沌系统可被分拆成两个子系统. Halle 等人 ${ }^{[4]}$ 提出可以不需 要分拆系统, 而实现系统同步, 但他们的方法由于确定同步区域的困难而局限于低维系统. Pyragas ${ }^{[5]}$ 提出连续反馈同步方法后, Yu 等人 ${ }^{[6]}$ 利用 Duffing 方程把这种同步方式应用到保密 通讯中, 但在他们的文章里, 假定信躁比(有用信号与混沌噪声的幅度比) 是很小的. 鉴于 Chua 电路在混沌同步通讯的重要地位, 本文着重利用 Chua 电路对连续反馈同步方式在保密 通讯中的应用作进一步研究. 研究发现: 应用连续反馈同步方式进行保密通讯时, 若有用信号 直接反馈到接收系统, 系统原来的同步特性发生改变, 接收系统实际上已变成一个参变耦合系 统. 引入恢复同步系数后, 我们发现当 $K_{\mathrm{r}}>2$ 时, 保密通讯得以实现, 并且与信躁比无关. 我 们还利用通用电路模拟器( PSPICE 软件) 做了仿真实验.

\section{1 原理与数值计算}

图 1 是一对间接耦合的 Chua 混沌电路用于保密通讯的系统方框图及电路图.

系统的动力学方程可写为 ${ }^{[8]}$

接收系统:

$$
\mid \begin{aligned}
& x>=\alpha[y-x-f(x)]+K[u+s(t)-x], \\
& y>=x-y+z, \\
& z>=-\beta y
\end{aligned}
$$

发射系统:

$$
\mid \begin{aligned}
& w>\alpha[v-u-f(u)] \\
& v>=u-v+w \\
& w>=-\beta v
\end{aligned}
$$

